\title{
Resolución de una necrosis lingual por leptospirosis canina
}

\author{
Koscinczuk, P.; Casco, J.J.; Cainzo, R.P.; Camoletto, D.A. \\ Cat. Patología Médica, Fac. Cs. Vet. Univ. Nac. Nordeste, \\ Cabral 2139, Corrientes, Argentina. E-mail: pkoscinczuk $a$, hotmail.com
}

\begin{abstract}
Resumen
Koscinczuk, P.; Casco, J.J.; Cainzo, R.P.; Camoletto, D.A.: Resolución de una necrosis lingual por leptospirosis canina. Rev. Vet. 32: 1, 114-116, 2021. Se describe la resolución clínica de una necrosis lingual asociada a insuficiencia renal aguda por leptospirosis. Un canino Boston terrier, macho entero de 4 años, a la exploración de la cavidad oral presentó alteraciones de la coloración, consistencia, sensibilidad y temperatura, así como ausencia de pulso en la punta de la lengua. En el perfil renal se observaron valores elevados de urea (183 $\mathrm{mg} / \mathrm{dl}$ ) y creatinina $(1,8 \mathrm{mg} / \mathrm{dl})$. Las pruebas de Ehrlichia y Leishmania fueron negativas, en tanto la prueba de microaglutinación microscópica, arrojó títulos de 1/3200 de los serovares Ballum castellonis y Canicola canicola de Leptospira interrogans. Los títulos elevados de anticuerpos y las manifestaciones clínicas compatibles, confirmaron el diagnóstico de daño renal agudo. Para el tratamiento se realizó fluidoterapia con Ringer lactato y dextosa al $5 \%$. Ante la sospecha de leptospirosis se administró penicilina con estreptomicina. Una vez estabilizada, se realizó una glosectomía parcial. Sin embargo, como la necrosis continuó avanzando, se decidió administrar $1 \mathrm{mg} / \mathrm{kg}$ de dexametasona por vía parenteral cada 12 horas, durante 3 días, y luego dosis decreciente. A los 8 días, el paciente estaba recuperado, aunque todavía poseía densidad urinaria isostenúrica. El valor de urea fue de $87 \mathrm{mg} / \mathrm{dl} \mathrm{y}$ el de creatinina 0,8 $\mathrm{mg} / \mathrm{dl}$, con un paciente recuperado. Si bien, los corticoides no se recomiendan ante un daño renal agudo, el uso de dexametasona estaría indicado para resolver el daño vascular.
\end{abstract}

Palabras clave: canino, glositis, necrosis, vasculitis, leptospirosis.

\begin{abstract}
Koscinczuk, P.; Casco, J.J.; Cainzo, R.P.; Camoletto, D.A. Resolution of a lingual necrosis for canine leptospirosis. Rev. Vet. 32: 1, 114-116, 2021. The present study describes the clinical resolution of a tongue necrosis associated whit an acute renal failure due to leptospirosis. A 4 year old male Boston terrier canine was present with a tongue that showed changes of color, consistency, sensibility and lack of pulse. Regarding the renal profile urea (183 mg/ dl) and creatinine $(1,8 \mathrm{mg} / \mathrm{dl})$, values were increased and micro agglutination technique gave titers 1/3200 of Leptospira interrogans serovars, Ballum castellonis y Canicola canicola. Increased antibody titles and clinical manifestations, confirmed the diagnosis of acute kidney damage. The treatment began with fluid therapy using Ringer lactato and dextrose $5 \%$. As leptospirosis was possible, antibiotic treatment was applied (penicillin combined with estreptomicina). Once the patient was stable, partial glosectomy was realized. Nevertheless, necrosis continued, so it was decided to use $1 \mathrm{mg} / \mathrm{kg}$ of dexametasona parenteraly during three days an then a lower dosis. Eight days later, the patient was recovered, although isostenuric urine density continued, urea $(87 \mathrm{mg} / \mathrm{dl})$ and creatinine $(0,8 \mathrm{mg} / \mathrm{dl})$ values decreased and the patient was stable. Despite corticosteroids are not recommended in acute renal failure in this case were used to control vasculitis. Considering the immune origin of vascular injury, treating the initial cause isn't enough, reason why immunosuppressive drugs are recommended.
\end{abstract}

Key words: canine, glossitis, necrosis, vasculitis, leptospirosis.

\section{INTRODUCCIÓN}

Un paciente canino macho entero, de 4 años, raza Boston Terrier, se presentó a la consulta clínica por una lesión en la lengua que le impedía comer y beber. Su estado general era malo, se encontraba letárgico, des- hidratado, anúrico e hipotérmico $\left(36,2^{\circ} \mathrm{C}\right)$. Según la anamnesis, había comenzado a decaer dos días antes.

Al abrir la boca, se pudo apreciar en la punta de la lengua una zona de color marrón verdoso (Figura 1). El límite entre el tejido necrótico y el tejido sano se encontraba delimitado por una línea congestiva. No se pudo registrar el pulso de la arteria sublingual, por lo cual se sospechó de una trombosis. 
El diagnóstico inicial fue de daño renal agudo, con congestión hepática asociada a una enfermedad infecciosa con lesión vascular o una trombosis que afectó la circulación lingual generando necrosis. En el urianálisis se observó una orina isostenúrica (1030) con proteinuria $(2+)$, la cual asociada a una deshidratación clínica del 10\% indicaría incapacidad de concentrar.

El valor inicial de transaminasas, urea y creatinina elevadas, confirmó la sospecha clínica inicial (Tabla 1). La etiología infecciosa se ratificó 15 días más tarde con los títulos elevados (1/3200) de Leptospira interrogans para los serovares Ballum castellonis y canicola.

\section{MATERIAL Y MÉTODOS}

Para la reposición inicial de fluidos se utilizaron cristaloides (solución de Ringer lactato y dextrosa al $5 \%$ en la proporción $3 / 1$ ).

Como la sospecha clínica de Leptospirosis era muy alta, se decidió comenzar el tratamiento con penicilinaestreptomicina $(22000 \mathrm{UI} / \mathrm{kg})$ vía parenteral cada 24 horas durante 3 días, para continuar con doxiciclina a dosis de $5 \mathrm{mg} / \mathrm{kg}$ vía oral cada 12 horas, durante 21 días.

\section{RESULTADOS}

A los ocho días el paciente se encontraba recuperado, con una orina isostenúrica, una urea aún elevada pero con una creatinina normal. Respecto a la resolución de la necrosis, una vez que el paciente estuvo hidratado, se realizó una intervención quirúrgica de exéresis de la porción necrótica.

La inducción anestésica se realizó con ketamina (5 $\mathrm{mg} / \mathrm{kg})$ y midazolam $(0,1 \mathrm{mg} / \mathrm{kg})$ vía parenteral. El límite de la zona de exéresis se determinó por la presencia de irrigación sanguínea. La porción necrótica correspondía aproximadamente al $40 \%$ de la lengua, sin embargo se extrajo un 50\% para asegurar un margen quirúrgico.

Realizada la hemostasia, se dejó abierta la herida para que se produzca la cicatrización por segunda intención. Cuatro horas después de la cirugía, con un paciente recuperado de la anestesia, se observó una progresión de la necrosis más allá de la línea de incisión quirúrgica (Figura 2). Fue en ese momento cuando se consideró el diagnóstico de vasculitis ${ }^{5}$ y se decidió tratarla con corticoides. Inicialmente se administró dexametasona ( $1 \mathrm{mg} / \mathrm{kg}$ vía endovenosa).

Luego de una hora, el paciente no solo había mejorado su estado de ánimo, sino que la lesión lingual dejó de progresar. Por este motivo se decidió seguir con la misma dosis de corticoide, cada 12 horas durante dos días. También se continuó con prednisolona $(1 \mathrm{mg} / \mathrm{kg}$ ) vía oral cada 12 horas, durante 3 días y luego, una dosis decreciente durante 2 semanas.

El paciente continúo recibiendo terapia de fluidos durante tres días más, durante los cuales se realizaron trabajos de reeducación para que pudiera comer y be-
Tabla 1. Parámetros observados en el paciente con daño renal agudo y hepatitis.

\begin{tabular}{lcc}
\hline parámetro & día 1 & día 8 \\
\hline densidad urinaria & 1030 & 1025 \\
proteinuria & $2+$ & neg \\
urea (mg/dl) & 183 & 878 \\
creatinina (mg/dl) & 1,8 & 0,8 \\
ALT (U/l) & 203 & s/d \\
AST $(\mathrm{U} / 1)$ & 218 & $\mathrm{~s} / \mathrm{d}$ \\
FAL $(\mathrm{U} / \mathrm{l})$ & 194 & $\mathrm{~s} / \mathrm{d}$ \\
\hline
\end{tabular}

ber por sus propios medios, optando por una dieta renal comercial.

En el control clínico realizado a los 8 días, los valores de urea y creatinina estaban dentro de rangos considerados como normales (Tabla 1). No obstante, se observaron áreas alopécicas en zonas del tórax, brazos y axilas. A los tres meses el paciente fue dado de alta con una orina hiperestenúrica (1045).

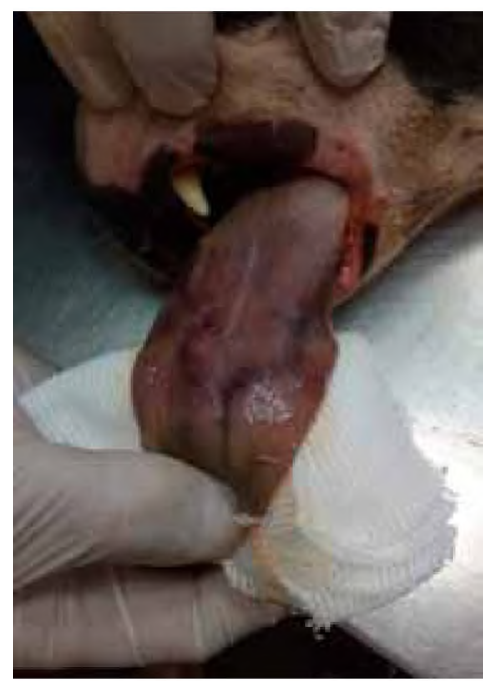

Figura 1. Necrosis lingual en el canino, donde se observa el límite del esfacelo.

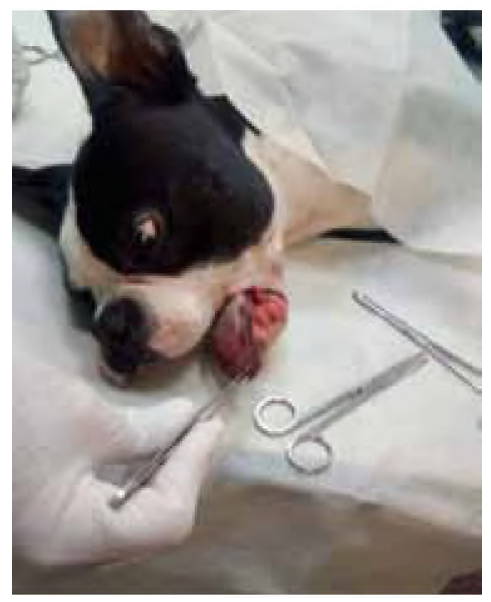

Figura 2. Resolución quirúrgica de la necrosis lingual, con progresión de la lesión 


\section{DISCUSIÓN}

La necrosis de la punta de lengua, no es una lesión frecuente, sin embargo cuando se presenta asociada a daño renal agudo debería considerarse la leptospirosis. Esta enfermedad es una de las zoonosis más extendida a nivel mundial y está causada principalmente por Leptospira interrogans ${ }^{6}$. En la década de 1990 se observó en perros un resurgimiento de serovares de leptospiras identificados como atípicas (Leptospira pomona, grippotyphosa, autumnalis, hardjo, bratislava y australis) $^{2}$.

La leptospirosis es un problema de salud pública mundial. La enfermedad clínica se caracteriza por disfunción renal que varía desde una lesión aguda leve a grave hasta una enfermedad renal crónica (ERC) que requiere diálisis ${ }^{6}$. Si bien se describe que hay una combinación de daño tubular agudo y nefritis intersticial, no es claro cuál es la lesión primaria ${ }^{1}$.

Se propone que la oligo-anuria es el resultado de una alteración del transporte tubular por la inhibición de la bomba de $\mathrm{Na}+/ \mathrm{K}+\mathrm{ATPase}^{6}$. Ello se acompaña de nefritis tubulointersticial, edema e infiltración mononuclear. A diferencia de otras enfermedades infecciosas, los cambios glomerulares son poco marcados ${ }^{6}$.

Por otra parte, en estos pacientes se puede observar disfunción multiorgánica y trombopatía ${ }^{6}$, por lo cual no es raro encontrar un aumento de las enzimas marcadoras de daño hepático e ictericia ${ }^{4}$. Otro hallazgo clínico tiene que ver con la trombocitopenia que ocurre asociada a la endotoxemia severa ${ }^{6} \mathrm{y}$ la vasculitis ${ }^{4}$.

En la leptospirosis canina, las fallas renales y hepáticas son potencialmente reversibles y deben tratarse lo antes posible y de manera agresiva. Lo primero que se debería considerar es mantener la hidratación y una producción de orina superior $2 \mathrm{ml} / \mathrm{kg} / \mathrm{h}$. Es importante considerar el uso de coloides o de furosemida en el caso de oligoanuria, evitando la sobrehidratación ${ }^{2}$.

El tratamiento etiológico implica el uso de antibióticos administrado generalmente en 2 fases: la ampicilina o la amoxicilina se pueden administrar por vía parenteral (20-25 mg / kg iv TID) durante la fase crítica inicial. Es importante tener en cuenta que los riñones eliminan estos fármacos y las concentraciones sanguíneas pueden volverse inapropiadamente altas en pacientes con disfunción renal.

Un método común para ajustar la cantidad de antibióticos es multiplicar la dosis normal por $1 /$ creatinina sérica ${ }^{2}$. En este caso se utilizó una dosis parenteral de penicilina con estreptomicina. Aún sabiendo que la estreptomicina tiene un impacto negativo sobre el riñón, se eligió esta combinación por su capacidad de controlar la leptospiuria.
A los tres días los pacientes continuaron con la dosis sugerida de doxiciclina $(10 \mathrm{mg} / \mathrm{kg}$ diariamente en 1 dosis), dado que es el antibiótico de elección y se prescribe durante un mínimo de 3 semanas para prevenir la diseminación renal persistente ${ }^{2,4}$

La necrosis de la lengua podría ser el resultado de una trombopatía o de una enfermedad inmuno-mediada como la vasculitis. Ambas situaciones están presentes en las infecciones por leptospirosis o por otras bacterias Gram negativas toxigénicas ${ }^{3,6}$. No obstante la resolución de una necrosis de lengua es quirúrgica, el tratamiento de la vasculitis requiere de la utilización de un corticoide ${ }^{3}$, de otra manera el daño vascular seguiría progresando.

Si bien se proponen dosis inmuno-supresoras altas, de 2 a $4 \mathrm{mg} / \mathrm{kg}$ de prednisolona, los autores consideraron comenzar con una dosis de dexamentasona de 1 $\mathrm{mg} / \mathrm{kg}$ dado el compromiso del riñón y siguiendo las recomendaciones de otros autores ${ }^{3}$.

La presentación clínica de una enfermedad aguda con fiebre, ictericia y daño renal agudo debería alertar al clínico de una posible leptospirosis. Sin embargo, cuando se presenta una necrosis lingual, la trombopatía y vasculitis deberían ser otro punto relevante a considerar para el tratamiento, ya que la activación de mediadores químicos pro-inflamatorios asociados a leptospirosis debería ser controlada.

\section{REFERENCIAS}

1. Bandeira CT, Athanazio DB, Stambovsky SA, Seguro AC. 2008. Renal involvement in leptospirosis; new insights into pathophysiology and treatment. The Braz Journal of Infect Dis 12: 248-252.

2. Gasken F. 2008. Leptospirosis canina. Actas del Congreso de la Asociación Mundial de Veterinaria de Pequeños Animales, Dublin, Irlanda, 2008

3. Innera M. 2013. Cutaneus vasculitis in small animals. Vet Clin Small Anim 43: 113-134

4. Sykes JE, Reagan KL. 2019. Diagnostic, treatment and management of Leptospirosis in dogs. Today's Veterinary Practice ep/oct; https://todaysveterinarypractice.com/diagno- sis-and-treatment-of-leptospirosis-in-dogs.

5. Swann JW, Priestnall SL, Dason C, Chang YM, Garden OA. 2015. Histologic and clinical features of primary and secondary vasculitis: a retrospective study of $42 \mathrm{dogs}$ (2004-2011). Journ Vet Diagn Investig 27: 489-496.

6. Yang C. 2007. Leptospirosis renal disease: understanding the initiation by tall-like receptors. Kidney Internat 72 : 918-925. 